\title{
Phylogenetic Analysis of Aedes aegypti Based on Mitochondrial ND4 Gene Sequences in Almadinah, Saudi Arabia
}

\author{
Khalil H. AL ALI 1*, Ayman A. El-Badry 2, Mouhanad AL ALI 3, Wael S.M. El-Sayed 4,5, Hesham A. \\ El-Beshbishy 1,6
}

${ }^{1}$ Department of Medical Laboratory Technology, College of Applied Medical Sciences, Taibah University, Almadinah Almanwra, Kingdom of Saudi Arabic

${ }^{2}$ Department of Medical Parasitology, Kasr Al-Ainy School of Medicine, Cairo University, Cairo, Egypt

${ }^{3}$ Department of Institut Supérieur de la Santé et des Bioproduits d'Angers, Université d'Angers, Angers, France

${ }^{4}$ Department of Microbiology, Faculty of Science, Ain Shams University, Cairo 11566, Egypt

${ }^{5}$ Department of Biology, Faculty of Science, Taibah University, Almadinah Almunawarah 344, Saudi Arabia

${ }^{6}$ Department of Biochemistry, Faculty of Pharmacy, Al-Azhar University, Nasr City, Cairo, Egypt

${ }^{*}$ Corresponding author: Khalil Al-Ali, Medical Laboratory Technology Department, College of Applied Medical Sciences, Taibah University, Almadinah Almanwra, Kingdom of Saudi Arabic. Tel: +966-148460008, Fax: +966-148475790, E-mail: kali@taibahu.edu.sa

Received: August 28, 2015; Revised: February 17, 2016; Accepted: February 24, 2016

Background: Aedes aegypti is the main vector of the yellow fever and dengue virus. This mosquito has become the major indirect cause of morbidity and mortality of the human worldwide. Dengue virus activity has been reported recently in the western areas of Saudi Arabia. There is no vaccine for dengue virus until now, and the control of the disease depends on the control of the vector.

Objectives: The present study has aimed to perform phylogenetic analysis of Aedes aegypti based on mitochondrial NADH dehydrogenase subunit 4 (ND4) gene at Almadinah, Saudi Arabia in order to get further insight into the epidemiology and transmission of this vector.

Materials and Methods: Mitochondrial ND4 gene was sequenced in the eight isolated Aedes aegypti mosquitoes from Almadinah, Saudi Arabia, sequences were aligned, and phylogenetic analysis were performed and compared with 54 sequences of Aedes reported in the previous studies from Mexico, Thailand, Brazil, and Africa.

Results: Our results suggest that increased gene flow among Aedes aegypti populations occurs between Africa and Saudi Arabia.

Conclusions: Phylogenetic relationship analysis showed two genetically distinct Aedes aegypti in Saudi Arabia derived from dual African ancestor.

Keywords: Aedes; Mosquito; ND4 gene; Phylogenetic; Saudi Arabia

\section{Background}

Aedes aegypti is the main vector responsible for transmission of the yellow fever, dengue virus, as well as, other arboviruses which affect human and numerous other animal species (1). It has become the major indirect cause of morbidity and mortality of human worldwide (2). According to the World Health Organization (WHO, 2007), it has been estimated that 50-100 million cases of the dengue fever occur every year in the world. Another recent study has estimated that 3900 million people, in 128 countries, are at risk of infection (3).

Dengue virus activity has been reported in the western areas of the Saudi Arabia; Jeddah, Makkah, and Almadinah $(4,5)$.

Until now, there is no vaccine for dengue virus, and the control of the disease mainly remains dependent on the control of the vector. This can explain why the rate of infection has recently increased dramatically. (6). Due to this reason, knowledge of vector dispersion has taken a prime importance and a critical role in controlling vector born disease.

In recent decades, entomologists have developed a number of morphological key characters for taxonomic goals in order to better understand the transmission and epidemiology of these vectors -borne diseases ( 7 , 
8). However, with advances in molecular biology, numerous studies have demonstrated that morphological keys are not sufficient. In addition, they are accompanied with several limitations (9) which might be due to several reasons, such as, minor genetic variation due to ecological impact, as well as, the constant use of insecticides, in addition to others factors (1012). Therefore, numerous molecular researches have been undertaken to find new molecular marker as an alternative tool to identify mosquito species.

\subsection{Phylogenetic Marker in Mosquitoes}

The idea of genetic marker is based on the principle that every species has a genetic identity which can be used as molecular marker for species identification. Several genetic markers have been studied in the previous studies, such as; ITS2 (Internal Transcribed Spacer), mitochondrial cytochrome c oxidase subunit $1(C O I)$ gene, and mitochocondrial NADH dehydrogenase subunit 4 (ND4) gene.

ITS2 has been proven to be a good molecular marker because of its highly conserved region and species specific sequence; as a result, it is largely being used as a phylogenetic marker for mosquito's taxonomy (13, 14).

COI-based DNA barcoding has been also used by Abigail Chan and co-workers to indentify mosquitoes in Singapore (15). This study has demonstrated that COI-based DNA barcoding can be also a good molecular marker for mosquitos' taxonomy.

However, mitochondrial NADH dehydrogenase subunit 4 (ND4) gene has gained increasing popularity in phylogenetic analysis and population genetic studies. (16) In addition, (ND4) gene has shown to be an excellent genetic marker $(6,17-20)$.

\section{Objectives}

Phylogenetic analysis regarding dengue vector Aedes aegypti is not assessed until now in Saudi Arabia based on the mitochondrial NADH dehydrogenase subunit 4 (ND4) gene. In this context, our study has aimed to construct phylogenetic tree of the Aedes aegypti mosquito based on ND4 gene to get more insight into the epidemiology and transmission of these vector born disease.

\section{Materials and Methods}

\subsection{Collection of Mosquito}

Eight Mosquitoes were collected from Almdinah in western part of the Saudi Arabia in 2013. Adult mos- quitoes specimens were collected using BG-sentinel traps (BioGents $\mathrm{AG}$, Germany), $\mathrm{CO}_{2}$ light traps, human baited net traps, and human landing catch method. Subsequently mosquitoes were killed through exposure to $-20^{\circ} \mathrm{C}$ for a few minutes, and were identified morphologically using the key described by Schaffner and co-workers (21). Following to the morphological identification, adults were stored frozen at $20^{\circ} \mathrm{C}$.

\subsection{DNA Extraction and Nad4 Sequencing}

Genomic DNA was extracted from the 8 adult mosquitoes using a QIA amp DNA Mini Kit and re-suspended the DNA in $80 \mu \mathrm{L}$ of buffer EB (from Qiagen) (22). Primers used to amplify the NADH dehydrogenase subunit 4 (ND4) gene were composed of ND4 forward-( $5^{\prime}$-TGATTGCCTAAGGCTCATGT- $\left.3^{\prime}\right)$ and ND4 reverse-(5'-TTCGGCTTCCTAGTCGTTCAT-3') (17). The polymerase chain reaction (PCR) amplification of the $344 \mathrm{pb}$ fragment was preceded by a five minute denaturation at $96^{\circ} \mathrm{C}$ and subsequent 35 cycles of the amplification consisted of $40 \mathrm{~s}$ at $94^{\circ} \mathrm{C}, 40 \mathrm{~s}$ at $56^{\circ} \mathrm{C}$ and $40 \mathrm{~s}$ at $72^{\circ} \mathrm{C}$, followed by a final extension step of five minutes at $72^{\circ} \mathrm{C}(6)$. PCR products were visualized on $1.5 \%$ agarose gels stained with GelRed (Biotium Ink., USA). Amplicons were gel-electrophoresed, excised from the gels and recovered with a QIAamp Gel Extraction Kit (Qiagen). Then, PCR products were sequenced in both directions using an automated MegaBACE 1000 Analysis System sequencer (GE Healthcare, UK).

\subsection{Phylogenetic Tree and Sequences Analysis}

Sequences obtained for mosquitoes were analyzed using BLAST program (http://blast.ncbi.nlm.nih.gov) to confirm the morphological identification. Mitochondrial ND4 gene sequences from eight Aedes aegypti were aligned using ClustalW software program (http://www.ebi.ac.uk/clustalw2/). phylogenetic trees were constructed by using MEGA software version 6 (23), to determine Phylogenetic relationships and Genetic variability.

Two phylogenetic trees were constructed for Aedes aegypti collected from Almadinah, Saudi Arabia. The first tree was based on the UPGMA algorithm within the Tamura-Nei genetic distance model. Bootstrap support was calculated by means of 1000 replicates. The Second tree was based on neighbor-joining (NJ) algorithm under the Tamura-Nei genetic distance model. Bootstrap support was calculated by means of 1000 replicates. 
All sequences were compared with 54 sequences available in the previous studies, from BrazilAY906835-AY906853 (20), JQ926718-JQ926719 (24), Mexico- JX297249- JX297259 (deposit in Gen Bank by Pfeiler et al. 2012), Thailand- JQ926720JQ926721 (24), and from Africa-JX427511JX427525 (25). Phylogenetic tree was constructed based on neighbor-joining (NJ) algorithm under the Tamura-Nei genetic distance model. Bootstrap support was calculated by means of 1000 replicates.

In this analysis Aedes koreicus Gen Bank accession number: KJ623732-KJ6237375 (deposit in GenBank by Raharimalala et al. 2014 (unpublished) were employed as out-group species. Information regarding sample size and localities are listed in the (Table 1).

\subsection{Statistical Analysis}

Statistical analyses were performed. The nucleotides diversity, Tajima's $D$ (26) and neutrality tests were calculated using MEGA software version 6.

\section{Results}

\subsection{DNA Extraction and Nad4 Sequencing}

In this study we report for the first time eight sequences of the Aedes aegypt collected from

Table 1. Localization and sample size of Aedes aegypti

\begin{tabular}{lcc}
\hline Ogranism & State & Sample size \\
\hline Aedes aegypti & Brazil & 21 \\
Aedes koreicus & Belgium & 6 \\
Aedes aegypti & Mexico & 11 \\
Aedes aegypti & Thailand & 2 \\
Aedes aegypti & Senegal & 14 \\
Aedes aegypti & Saudi arabia & 8 \\
\hline
\end{tabular}

Table 2. Fragments' length and GenBank accession Number of Aedes aegypt collected from Almadinah, Saudi Arabia

\begin{tabular}{lcccc}
\hline Ogranism & $\begin{array}{c}\text { Gen Bank } \\
\text { accession } \\
\text { Number }\end{array}$ & $\begin{array}{c}\text { Length } \\
\mathbf{( p b )}\end{array}$ & City & state \\
\hline Aedes aegypti & $\mathrm{AB} 594491.1$ & $344 \mathrm{bp}$ & $\mathrm{Al}$-Madinah Saudi arabia \\
Aedes aegypti & $\mathrm{AB} 594490.1$ & $344 \mathrm{bp}$ & $\mathrm{Al}$-Madinah Saudi arabia \\
Aedes aegypti & $\mathrm{AB} 594489.1$ & $344 \mathrm{bp}$ & $\mathrm{Al}$-Madinah Saudi arabia \\
Aedes aegypti & $\mathrm{AB} 594488.1$ & $344 \mathrm{bp}$ & $\mathrm{Al}$-Madinah Saudi arabia \\
Aedes aegypti & $\mathrm{AB} 594487.1$ & $344 \mathrm{bp}$ & $\mathrm{Al}$-Madinah Saudi arabia \\
Aedes aegypti & $\mathrm{AB} 594486.1$ & $344 \mathrm{bp}$ & $\mathrm{Al}$-Madinah Saudi arabia \\
Aedes aegypti & $\mathrm{AB} 594485.1$ & $344 \mathrm{bp}$ & $\mathrm{Al}$-Madinah Saudi arabia \\
Aedes aegypti & $\mathrm{AB} 594484.1$ & $344 \mathrm{bp}$ & $\mathrm{Al}$-Madinah Saudi arabia \\
\hline
\end{tabular}

Almadinah, Saudi arabia (KSA). The eight sequences were deposited in the National Center for Biotechnology Information (NCBI) in 2010 GenBank accession number are listed in (Table 2).

\subsection{Phylogenetic Tree and Sequences Analysis}

The obtained sequences were analyzed using BLAST program to confirm morphological identification, all sequences show high similarities with NADH dehydrogenase subunit 4 (ND4) gene with an identity of $100 \%$ and E-value: $8 \mathrm{e}-179$.

Two phylogenetic trees were constructed for Aedes aegypti collected from Almadinah, Saudi Arabia by using two different algorithm UPGMA and neighborjoining (NJ) algorithm (Figure 1).

The results obtained from the two models indicate that Aedes aegypti S2, S3, S4, S5, S6, and S7 share high similarity and form one group, whereas, this
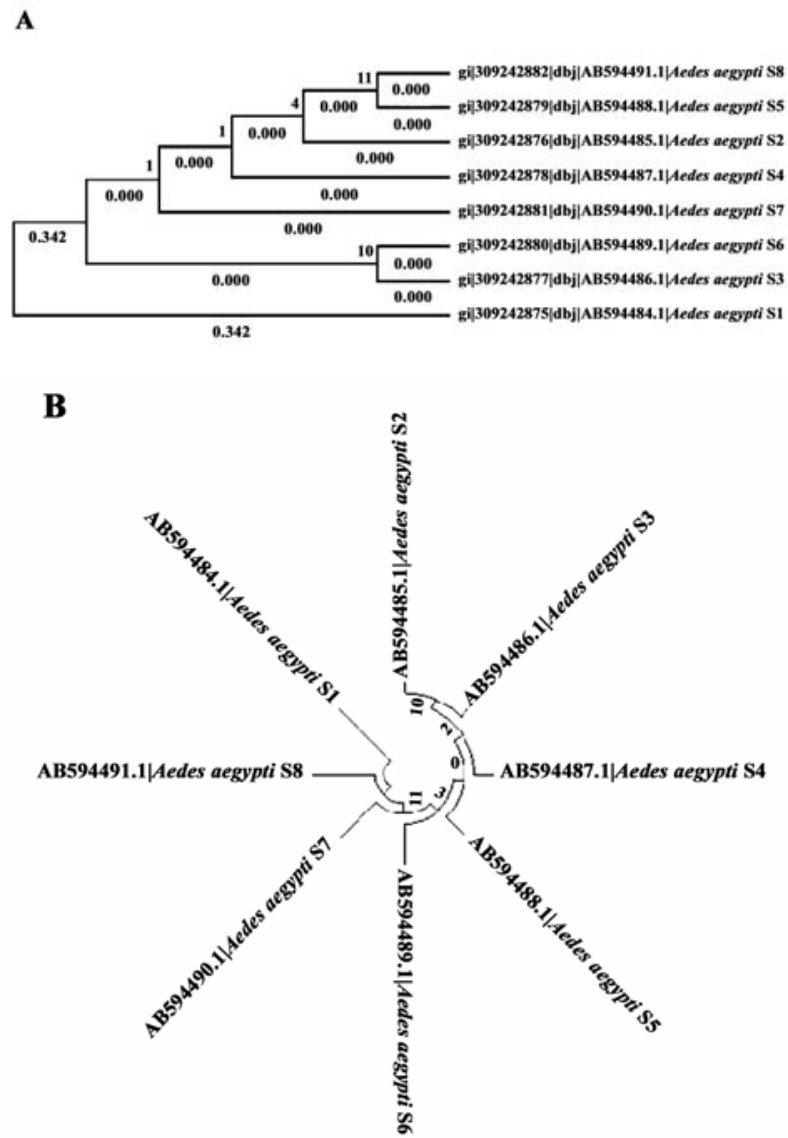

Figure 1. Phylogenetic relationships among Aedes aegypt collected from Almadinah, based on the UPGMA method under the Tamura-Nei genetic distance model. A: Bootstrap values are marked on the branches. B: Phylogenetic relationships among Aedes aegypt collected from Almadinah, based on the neighborjoining (NJ) algorithm under the Tamura-Nei genetic distance model. Bootstrap values are marked on the branches 
group shares low similarity with $\mathrm{S} 1$. All sequences have been compared with 54 sequences available from previous studies. Phylogenetic tree was constructed based on neighbor-joining (NJ) algorithm under the Tamura-Nei genetic distance model. Bootstrap support was calculated by means of 1000 replicates.

The results indicate that among 54 Aedes located in different states, the group consisting of Aedes aegypti S2, S3, S4, S5, S6, and S7 show close relationship to the Aedes aegypti located in Africa, as well as, Aedes agypti located in Mexico, On the contrary, the aedes aegypti $\mathrm{S} 1$ is disclosed to the group ( $22, \mathrm{~S} 3, \mathrm{~S} 4, \mathrm{~S} 5$, $\mathrm{S} 6$, and S7) and is very close to a different group of Aedes aegypti located in Africa (Moore et al. 2013). (Figure 2).

Statistical analyses were performed and genetic diversity was calculated. The highest value of the nucleotide diversity was found in this study compared to the previous studies (Table 3 ).

\section{Discussion}

Our results demonstrate that among the eight Aedes

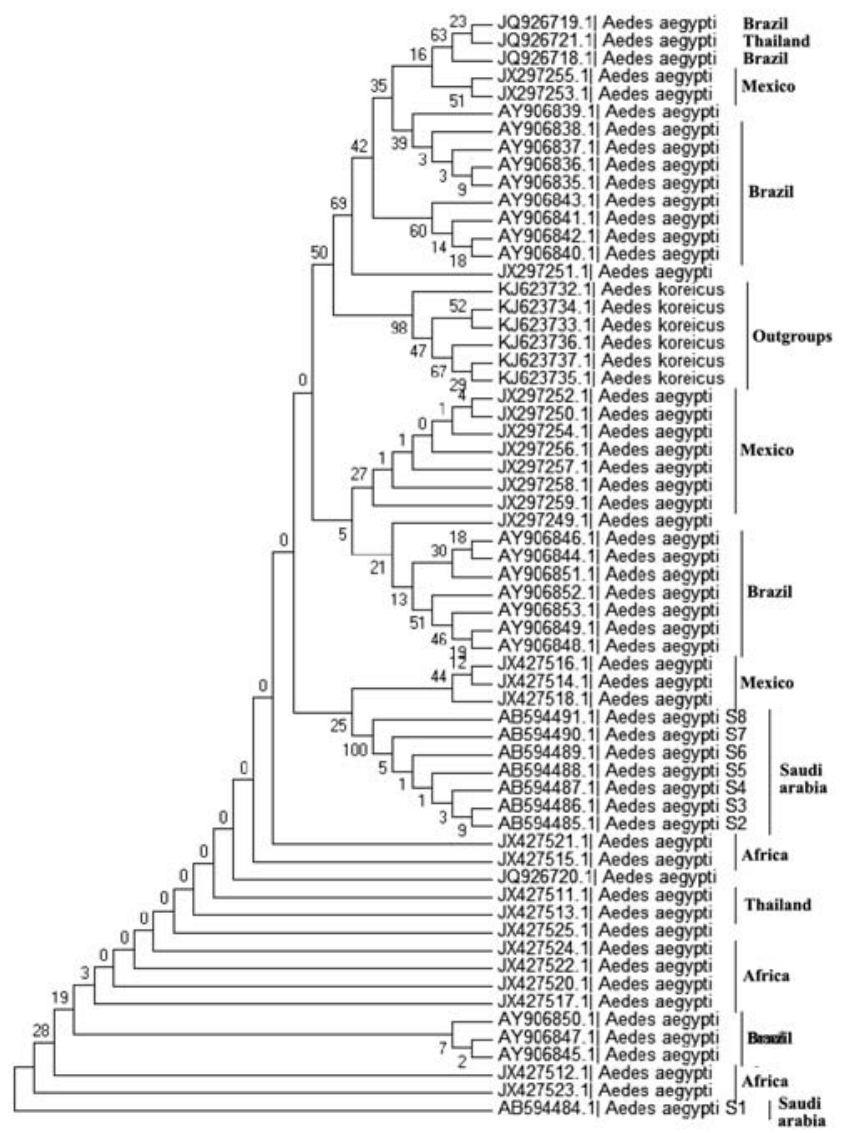

Figure 2. Phylogenetic relationships among Aedes aegypt, based on the neighbor-joining (NJ) algorithm under Tamura-Nei genetic distance model. Bootstrap values are marked on the branches
Table 3. Genetic variability and neutrality tests of the ten Aedes aegypti samples from Amadinah, Saudi arabia

\begin{tabular}{cccccc}
\hline $\boldsymbol{m}$ & $\boldsymbol{S}$ & $\boldsymbol{P}_{\boldsymbol{s}}$ & $\boldsymbol{\theta}$ & $\boldsymbol{\pi}$ & $\boldsymbol{D}$ \\
\hline 8 & 149 & 0.438235 & 0.169016 & 0.109559 & -1.921827 \\
\hline $\begin{array}{l}m=\text { number of sequences, } s=\text { number of segregating sites, } P_{s}=\mathrm{SIN}, \theta=\mathrm{Ps} / \\
\text { a1, } \pi=\text { nucleotide diversity, and } D \text { is the Tajima test statistic }\end{array}$
\end{tabular}

aegypti collected from Almadina in Saudi Arabia, only seven Aedes have shared high genetic similarity and form one unique group consisting of S2, S3, S4, S5, $\mathrm{S} 6, \mathrm{~S} 7$, and $\mathrm{S} 8$.

In fact, this group discloses and shares low similarity with $\mathrm{S} 1$, which is an Aedes aegypti collected from the same city (Almadinah) at the same time in 2010.

In 2013, Moor and co-workers have studied Aedes aegypti populations from Senegal, West Africa, Kenya, and East Africa based on mitochondrial DNA analysis. Phylogenetic analyses have shown that population of Aedes aegypti out of Africa are consisted of mosquitoes that have arisen from one of the two ancestral clades. One clade is associated with the West Africa, while the second has its origin from the first and contains primarily mosquitoes from East Africa.

Unexpectedly, our study shows that Aedes aegypti $\mathrm{S} 1$ discloses a close relationship with the first ancestral clade, which is associated with the West Africa. Whereas, the second African ancestor, which is associated with the East Africa and arises from the first one, shows a close relationship with Aedes group consisting of S2, S3, S4, S5, S6, S7, and S8. However, compiling our results with those described previously by Moor et al. (2013), we can state that Aedes group consisting of S2, S3, S4, S5, S6, S7, and S8 are derived from Aedes S1.

These results suggest that the main vector of yellow fever and dengue virus might have been introduced into Saudi Arabia by African pilgrims and continued to circulate in western Saudi Arabia. Our finding support the results of (Esam et al. 2015), as well as, the hypothesis of the dual African Origins for Aedes aegypti (Moore et al. 2013).

\section{Conclusions}

As a conclusion, our results suggest that the increased gene flow among Aedes aegypti populations occurs between Africa and Saudi Arabia. Phylogenetic relationship analysis shows that the two genetically distinct Aedes aegypti in Saudi Arabia have deriven their origin from dual African ancestors. Further supports to our finding come from the fact that commer- 
cial exchanges and transports from dengue endemic regions, in addition to foreign pilgrims could play a crucial role in the disease transmission.

Finally, we can express that, it is difficult to determine whether the observed genetic distance in Saudi Arabia Aedes population is related to geographic distance, or other factors respectively.

\section{References}

1. Forattini O. Culicidologia médica, vol. 2: Identificação, biologia, epidemiologia. São Paulo: Editora da Universidade de São Paulo. 2002

2. Taraphdar D, Sarkar A, Chatterjee S. Mass scale screening of common arboviral infections by an affordable, cost effective RT-PCR method. Asian Pac J Trop Biomed. 2012;2(2):97-101. DOI: 10.1016/S2221-1691(11)60200-1

3. Brady OJ, Gething PW, Bhatt S, Messina JP, Brownstein JS, Hoen AG, et al. Refining the global spatial limits of dengue virus transmission by evidence-based consensus. PLoS Negl Trop Dis. 2012;6(8):e1760. DOI: 10.1371/journal.pntd.000 1760

4. El-Badry AA, El-Beshbishy HA, Al-Ali KH, Al-Hejin AM, ElSayed WSM. Molecular and seroprevalence of imported dengue virus infection in Al-Madinah, Saudi Arabia. Comp Clin Path. 2013;23(4):861-868. DOI: 10.1007/s00580-013-1704-x

5. Azhar EI, Hashem AM, El-Kafrawy SA, Abol-Ela S, AbdAlla AM, Sohrab SS, et al. Complete genome sequencing and phylogenetic analysis of dengue type 1 virus isolated from Jeddah, Saudi Arabia. Virol J. 2015;12:1. DOI: 10.1186/s 12985-014-0235-7

6. Bosio CF, Harrington LC, Jones JW, Sithiprasasna R, Norris DE, Scott TW. Genetic structure of Aedes aegypti populations in Thailand using mitochondrial DNA. Am J Trop Med Hyg. 2005;72(4):434-442.

7. Rattanarithikul R, Harrison BA, Panthusiri P, Coleman RE. Illustrated keys to the mosquitoes of Thailand I. Background; geographic distribution; lists of genera, subgenera, and species; and a key to the genera. Southeast Asian J Trop Med Public Health. 2005;36(1):1-80.

8. Rattanarithikul R, Harbach RE, Harrison BA, Panthusiri P, Coleman RE, Richardson JH. Illustrated keys to the mosquitoes of Thailand. VI. Tribe Aedini. Southeast Asian J Trop Med Public Health. 2010;41(1):1-225.

9. Jinbo U, Kato T, Ito M. Current progress in DNA barcoding and future implications for entomology. $J$ Entomol Sci. 2011;14(2):107-124. DOI: 10.1111/j.1479-8298.2011.00449 .x

10. Huber K, Le Loan L, Hoang TH, Ravel S, Rodhain F, Failloux AB. Genetic differentiation of the dengue vector, Aedes aegypti (Ho Chi Minh City, Vietnam) using microsatellite markers. Mol Ecol. 2002;11(9):1629-1635. DOI: 10.1046/j. 1365-294X.2002.01555.X

11. Paupy C, Chantha N, Reynes JM, Failloux AB. Factors influencing the population structure of Aedes aegypti from the main cities in Cambodia. Heredity 2005;95(2):144-147. DOI: 10.1038/sj.hdy.6800698

12. Scarpassa VM, Cardoza TB, Cardoso Junior RP. Population genetics and phylogeography of Aedes aegypti (Diptera: Culicidae) from Brazil. Am J Trop Med Hyg. 2008;78(6):895-903.

13. Wesson DM, Porter CH, Collins FH. Sequence and secondary structure comparisons of ITS rDNA in mosquitoes (Diptera: Culicidae). Mol Phylogenet Evol. 1992;1(4):253-269. DOI: 10.1016/1055-7903(92)90001-W

14. Marrelli MT, Sallum MA, Marinotti O. The second internal transcribed spacer of nuclear ribosomal DNA as a tool for Latin American anopheline taxonomy-a critical review. Mem Inst Oswaldo Cruz. 2006;101(8):817-832. DOI: http://dx.doi. org/10.1590/S0074-02762006000800002

15. Chan A, Chiang LP, Hapuarachchi HC, Tan CH, Pang SC, Lee $\mathrm{R}$, et al. DNA barcoding: complementing morphological identification of mosquito species in Singapore. Parasit Vectors. 2014;7:569. DOI: 10.1186/s13071-014-0569-4

16. Urdaneta-Marquez L, Bosio C, Herrera F, Rubio-Palis Y, Salasek M, Black WCt. Genetic relationships among Aedes aegypti collections in Venezuela as determined by mitochondrial DNA variation and nuclear single nucleotide polymorphisms. Am J Trop Med Hyg. 2008;78(3):479-491.

17. Seixas G, Salgueiro P, Silva AC, Campos M, Spenassatto C, Reyes-Lugo M, et al. Aedes aegypti on Madeira Island (Portugal): genetic variation of a recently introduced dengue vector. Mem Inst Oswaldo Cruz. 2013;108(1):3-10. DOI: http: //dx.doi.org/10.1590/0074-0276130386

18. Da Costa-Da-Silva AL, Capurro ML, Bracco JE. Genetic lineages in the yellow fever mosquito Aedes (Stegomyia) aegypti (Diptera: Culicidae) from Peru. Mem Inst Oswaldo Cruz. 2005;100(6):539-544. DOI: http://dx.doi.org/10.1590/S007402762005000600007

19. Bracco JE, Capurro ML, Lourenco-de-Oliveira R, Sallum MA. Genetic variability of Aedes aegypti in the Americas using a mitochondrial gene: evidence of multiple introductions. Mem Inst Oswaldo Cruz. 2007;102(5):573-580. DOI: http://dx. doi.org/10.1590/S0074-02762007005000062

20. Paduan Kdos S, Ribolla PE. Mitochondrial DNA polymorphism and heteroplasmy in populations of Aedes aegypti in Brazil. J Med Entomol. 2008;45(1):59-67.

21. Schaffner F. Les moustiques d'Europe logiciel d'identification et d'enseignement; an identification and training programme; francais, english $=$ The mosquitoes of Europe. Paris: IRD Éditions; 2001

22. Collins FH, Mendez MA, Rasmussen MO, Mehaffey PC, Besansky NJ, Finnerty V. A ribosomal RNA gene probe differentiates member species of the Anopheles gambiae complex. Am J Trop Med Hyg. 1987;37(1):37-41.

23. Kumar S, Tamura K, Nei M. MEGA3: Integrated software for Molecular Evolutionary Genetics Analysis and sequence alignment. Brief Bioinform. 2004;5(2):150-163. DOI: 10. 1093/bib/5.2.150

24. Paupy C, Le Goff G, Brengues C, Guerra M, Revollo J, Barja Simon Z, et al. Genetic structure and phylogeography of Aedes aegypti, the dengue and yellow-fever mosquito vector in Bolivia. Infection, genetics and evolution. Infect Genet Evol. 2012;12(6):1260-1269. DOI: 10.1016/j.meegid.2012.04.012

25. Moore M, Sylla M, Goss L, Burugu MW, Sang R, Kamau LW, et al. Dual African origins of global Aedes aegypti s.l. populations revealed by mitochondrial DNA. PLoS Negl Trop Dis. 2013;7(4):e2175. DOI: 10.1371/journal.pntd.0002175

26. Tajima F. Statistical method for testing the neutral mutation hypothesis by DNA polymorphism. Genetics 1989;123(3):585-595. 